1 Universidade de São Paulo (USP), Faculdade de Medicina de Ribeirão Preto, Departamento de Medicina Social - Ribeirão Preto (SP), Brasil. joserodrigues.saude@gmail. com

2 Universidade do Estado do Rio de Janeiro (Uerj), Instituto de Medicina Social - Rio de Janeiro (RJ), Brasil.

3 Universidade Federal do Rio Grande do Norte (UFRN), Escola Multicampi de Ciências Médicas Caicó (RN), Brasil.

\section{Educação interprofissional e as ações formativas do eixo do provimento emergencial do Programa Mais Médicos}

\author{
Interprofessional education and the formative actions of the axis of \\ emergency provision of the More Doctors Program
}

José Rodrigues Freire Filho', Carinne Magnago ${ }^{\mathbf{2}}$, Marcelo Viana da Costa ${ }^{\mathbf{3}}$, Aldaísa Cassanho

Forster ${ }^{1}$

DOI: $10.1590 / 0103-11042019 S 104$

RESUMO Este artigo objetivou identificar se as ações formativas do eixo do provimento emergencial do Programa Mais Médicos se apoiam em elementos teórico-metodológicos da educação interprofissional, na perspectiva dos participantes do programa. Trata-se de estudo exploratório, desenvolvido em 2016, em Minas Gerais. Os dados foram coletados mediante entrevistas individuais $(n=30)$ e nove grupos focais $(n=52)$ com participantes do programa, e tratados por análise de conteúdo, que produziu três categorias: Os pressupostos da educação interprofissional enunciados nos ciclos formativos do Programa Mais Médicos; As mudanças no processo de trabalho em equipe, na perspectiva dos elementos da educação interprofissional, desencadeadas pelos ciclos formativos; e Dificuldades para a abordagem da educação interprofissional nos ciclos formativos. Entre os elementos enunciados nos processos formativos, estão o aprendizado sobre o papel de cada profissão, o compartilhamento de experiências e a centralidade do cuidado no paciente. Por outra via, o preceito fundamental da educação interprofissional de aprendizado entre duas ou mais profissões não é aplicado sistematicamente. O Mais Médicos se constitui política propícia para o fortalecimento da interprofissionalidade, especialmente porque sua proposta se assenta na lógica da educação permanente, que demanda o estabelecimento de uma relação de mútua influência entre a educação e o trabalho em saúde.

PALAVRAS-CHAVE Consórcios de saúde. Relações interprofissionais. Equipe de assistência ao paciente. Estratégia Saúde da Família. Educação médica.

ABSTRACT This article aimed to identify if the formative actions of the axis the emergency provision of the More Doctors Program are based on theoretical and methodological elements of interprofessional education from the perspective of the participants of the program. It is an exploratory study, developed in 2016, in Brazil. Data were collected through individual interviews $(n=30)$ and nine focus groups $(n=52)$, and treated by content analysis, which produced three categories: Assumptions of interprofessional education set forth in the formative cycles of the More Doctors; Changes in the process of teamwork, from the perspective of the elements of interprofessional education, triggered by the formative cycles; Difficulties for the approach of interprofessional education in the formative cycles. Among the elements enunciated 
in the formative processes are the learning about the role of each profession, the sharing of experiences, and the centrality of care in the patient. However, the fundamental precept of interprofessional education of learning between two or more professions is not applied. The More Doctors constitutes a propitious policy for the strengthening of interprofessionality, because its proposal is based on the logic of permanent education, which demands the establishment of a relationship of mutual influence between education and work in health.

KEYWORDS Health consortia. Interprofessional relations. Patient care team. Family Health Strategy. Education, medical.

\section{Introdução}

O processo de fortalecimento e consolidação do Sistema Único de Saúde (SUS), a partir dos princípios de universalidade e integralidade, tem encontrado importantes referenciais que sustentam a necessidade de reformas na produção dos serviços de saúde baseadas no trabalho colaborativo em equipe, com vistas a garantir a centralidade do usuário no processo do cuidado em saúde.

Na mesma direção, recomendações mundiais apontam a necessidade de maiores esforços no sentido de fortalecer a colaboração profissional, tendo como referencial a abordagem da Educação Interprofissional em Saúde (EIP) $)^{\mathbf{1} \mathbf{2}}$.

No Brasil, no entanto, ainda são escassos os estudos que versam sobre esse tema, que ganhou notoriedade a partir de 2010 após duas publicações apontarem a EIP como elemento promotor de uma necessária reforma no modelo de formação profissional em saúde ${ }^{\mathbf{1 , 3}}$.

A literatura traz importantes contribuições no sentido de definir a EIP, marcando a sua intencionalidade de melhorar a capacidade de colaboração e, consequentemente, os resultados da atenção à saúde. O Centro para o Avanço da Educação Interprofissional, Centre for the Advancement of Interprofessional Education (Caipe), no Reino Unido, apresenta a definição mais amplamente socializada na literatura internacional, também compartilhada pela
Organização Mundial da Saúde, que defende a EIP como a ocasião em que duas ou mais profissões da área da saúde aprendem com, a partir de e sobre as outras para melhorar a colaboração profissional e a qualidade do cuidado ${ }^{\mathbf{1 , 4}}$.

No cenário brasileiro, ao discutir a abordagem da EIP e possibilidades de sua incorporação na formação em saúde, merece destaque a instituição do Programa Mais Médicos (PMM), sendo uma das mais importantes estratégias de redução das desigualdades de oferta e distribuição de cursos de graduação de medicina e de profissionais médicos no território brasileiro. Ao incluir, em sua proposta, a reestruturação da formação médica do País, o PMM constitui-se como campo propício para adoção de iniciativas indutoras da EIP e prática colaborativa.

Nessa linha, o eixo do programa relacionado com a formação médica apresenta como um de seus resultados a publicação de novas Diretrizes Curriculares Nacionais para os cursos de graduação em medicina, instituídas em $2014^{5}$, as quais apontam que o graduando em medicina deverá aprender interprofissionalmente, a partir da troca de saberes com profissionais da saúde, estimulando o aprimoramento da colaboração. Ademais, estimula a aprendizagem compartilhada, a comunicação interprofissional e as iniciativas que valorizem os usuários como sujeito central da produção dos serviços de saúde ${ }^{6}$. 
O eixo do provimento emergencial de médicos para atuação em locais de maior necessidade e vulnerabilidade, por sua vez, dispõe que os médicos do PMM sejam submetidos a processos formativos, com a finalidade de aprimorar a prática da medicina de família e comunidade consoante ao modelo de atenção à saúde do Brasil.

O escopo das atividades pedagógicas previstas estrutura-se em dois ciclos formativos. O primeiro contempla um Curso de Especialização em Saúde da Família ofertado por uma instituição de ensino superior integrante do Sistema Universidade Aberta do SUS (UNA-SUS); enquanto o segundo prevê um conjunto de atividades educacionais no nível de aperfeiçoamento e extensão, que objetivam aprofundar o conhecimento em temas relevantes para atuação na atenção básica. No âmbito desses dois ciclos, há a figura do tutor, responsável pela coordenação e indicação das atividades a serem executadas pelos médicos e supervisores; e há o supervisor acadêmico, que acompanha as atividades dos médicos in loco.

Sobre o segundo eixo do PMM, uma investigação dos Projetos Políticos Pedagógicos (PPP) dos cursos de especialização ofertados no primeiro ciclo de formação apontou que, embora nenhum dos PPP explicite a interprofissionalidade como base pedagógica, eles apresentam algum grau de correspondência com os elementos da EIP. Ademais, convoca a realização de novos estudos que, entre outros recursos, dê voz aos diferentes atores imbricados nos processos formativos do PMM, dado que o previsto documentalmente não necessariamente é de fato operacionalizado ${ }^{7}$.

Com base no contexto apresentado e no entendimento de que, além da especialização, os processos de aperfeiçoamento, que utilizam a realidade do serviço como recurso pedagógico, representam importante oportunidade para $o$ desenvolvimento de competências interprofissionais, questiona-se: o conjunto de ações formativas previstas no eixo do provimento emergencial do PMM se alinha aos marcos teóricos e metodológicos da EIP para promoção de mudanças nas práticas profissionais em direção ao desenvolvimento da colaboração interprofissional em saúde? Para responder a esta questão, este estudo tem como objetivo identificar se as ações formativas do eixo do provimento emergencial do PMM se apoiam em elementos teóricos e metodológicos da EIP, na perspectiva dos participantes do programa.

\section{Métodos}

Trata-se de estudo exploratório, transversal, de abordagem qualitativa, desenvolvido em novembro e dezembro de 2016.

O cenário de pesquisa foi o território sob atuação de quatro das cinco instituições supervisoras do provimento emergencial do PMM no estado de Minas Gerais: Região Central - Secretaria Municipal de Saúde de Belo Horizonte (SMSA-BH); Norte - Universidade Estadual de Montes Claros (Unimontes); Vale do Jequitinhonha - Universidade Federal dos Vales do Jequitinhonha e Mucuri (UFVJM); e Triângulo Mineiro - Universidade Federal de Uberlândia (UFU). Essas instituições são responsáveis pela supervisão de $52 \%(n=448)$ do total de médicos alocados via PMM no estado.

Considerando o tamanho do território, optou-se por selecionar um conjunto de municípios para o desenvolvimento deste estudo: Belo Horizonte, Montes Claros e Uberlândia, sob supervisão da SMSA-BH, Unimontes e UFU, respectivamente; e Diamantina e Couto Magalhães, sob supervisão da UFVJM. A escolha desses municípios se deu em razão de eles fazerem parte do território de atuação do autor principal, no período em que os dados foram coletados.

A população da pesquisa foi constituída pelos médicos, tutores, supervisores e profissionais das equipes de atenção básica, às quais os médicos participantes do projeto estão vinculados.

Para determinação da amostra representativa do território, buscou-se incluir três equipes da Estratégia Saúde da Família (ESF) por instituição supervisora: uma equipe com 
médicos de perfil brasileiro, isto é, médicos de nacionalidade e formação brasileiras; uma equipe com médicos de perfil intercambista; e uma equipe com médicos advindos da cooperação estabelecida entre a Organização Pan-Americana da Saúde (Opas) e Cuba.

Em se tratando de pesquisa envolvendo seres humanos, em obediência às diretrizes da Resolução no 466 , de 12 de dezembro de 2012, do Conselho Nacional de Saúde, o projeto foi submetido ao Comitê de Ética em Pesquisa da Universidade de São Paulo e aprovado sob o Parecer $\mathrm{n}^{0}$ 1.264.019.

Para a seleção dos envolvidos na pesquisa, foram adotados os seguintes critérios de inclusão: (1) para os médicos: ser vinculado ao PMM pelo eixo do provimento emergencial e ter realizado minimamente $\mathrm{o} 1^{\circ}$ ciclo formativo do projeto (Curso de Especialização em Saúde da Família); (2) para os profissionais: ser profissional da saúde que atua diretamente com o médico do PMM, preferencialmente, mas não exclusivamente, componente da equipe mínima de ESF (enfermeiro, técnico/auxiliar de enfermagem e Agente Comunitário de Saúde - ACS), com tempo mínimo de atuação na ESF de um ano; (3) para os tutores e supervisores: estar vinculado a uma das instituições supervisoras escolhidas por este estudo há, no mínimo, um ano.

O tempo mínimo de um ano para a atuação dos profissionais, tutores e supervisores foi determinado por acreditar que ele seja suficiente para conhecer e se apropriar do cotidiano do trabalho em equipe na ESF.

Os critérios de exclusão delimitados foram: não participação da discussão durante os grupos focais ou solicitação para se retirar da pesquisa após a coleta dos dados. Nenhum desses critérios precisou ser aplicado.

A amostra final totalizou 82 sujeitos, sendo 52 profissionais de saúde integrantes das equipes de saúde da família, entre enfermeiros, técnicos e auxiliares de enfermagem, ACS e assistente social, 12 supervisores, 11 médicos e 7 tutores.

Os dados foram coletados por meio de entrevista em profundidade, aplicada aos médicos, tutores e supervisores, e grupo focal, realizado com os demais integrantes das equipes de saúde da família. Cumpre esclarecer que foi necessária apenas uma participação de cada voluntário nos momentos de coleta de dados.

As entrevistas foram operacionalizadas com auxílio de roteiro não estruturado, composto por sete questões relacionadas com os ciclos formativos do eixo do provimento emergencial do PMM e processo de trabalho na ESF com foco na abordagem da EIP. Os grupos focais, por sua vez, foram conduzidos por roteiro com quatro questões disparadoras pertinentes aos processos de trabalho das equipes.

Foram realizadas 30 entrevistas e 9 grupos focais com tempos médios de duração de 58 e 93 minutos respectivamente. Todos os depoimentos foram registrados em equipamento de áudio digital. Os dados foram tratados com base na proposta de análise de conteúdo, que pressupõe três fases: pré-análise; exploração; e análise e inferência ${ }^{8}$.

Na pré-análise, realizou-se a transcrição integral das entrevistas e grupos focais, dando origem ao corpus analítico, seguida da leitura flutuante do material. A etapa de exploração do material teve por objetivo destacar os recortes de ordem semântica do corpus, que expressassem elementos da EIP previstos nas diretrizes propostas por Barr ${ }^{9}$ para construção e avaliação de contextos educacionais na perspectiva da EIP.

Dessa forma, o corpus analítico foi composto pelos seguintes recortes: aprendizado conjunto entre duas ou mais profissões; desenvolvimento de competências para a prática colaborativa; aprendizado sobre o papel de cada profissão; a colaboração profissional como estratégia de melhoria da qualidade da atenção; aprendizagem interprofissional como estratégia pedagógica de integração curricular; utilização de recursos pedagógicos que promovam o compartilhamento entre diferentes profissões e que usem o contexto do trabalho como disparador da aprendizagem; aprendizagem baseada na inclusão e centralização do usuário e na participação efetiva dos profissionais do 
serviço nas práticas educativas. Esses recortes foram agrupados por similaridade em três categorias e suas respectivas subcategorias.

Outrossim, na fase de inferência e interpretação, as informações foram condensadas e destacadas, possibilitando interpretações e análise crítica do material. Em seguida, para garantia do anonimato dos participantes do grupo, as falas sistematizadas foram identificadas apenas pela inserção do participante no PMM: médico, tutor, preceptor ou equipe de ESF a que pertence o sujeito da pesquisa.

\section{Resultados}

A análise dos depoimentos evidenciou três categorias temáticas: Os pressupostos da EIP enunciados nos ciclos formativos do PMM; As mudanças no processo de trabalho em equipe, na perspectiva dos elementos da EIP, desencadeadas pelos ciclos formativos; e Dificuldades para a abordagem da EIP nos ciclos formativos. Elas são elencadas, com suas respectivas subcategorias, no quadro 1 .

Quadro 1. Categorias e subcategorias elaboradas a partir do corpus da pesquisa. Minas Gerais, 2016

\begin{tabular}{|c|c|}
\hline Categorias & Subcategorias \\
\hline \multirow{4}{*}{$\begin{array}{l}\text { Os pressupostos da EIP enunciados nos } \\
\text { ciclos formativos do PMM }\end{array}$} & Aprendizado sobre o papel de cada profissão \\
\hline & $\begin{array}{l}\text { A colaboração profissional como estratégia de melhoria da qualidade da } \\
\text { atenção }\end{array}$ \\
\hline & O estímulo às práticas profissionais colaborativas \\
\hline & $\begin{array}{l}\text { A aprendizagem compartilhada baseada nas necessidades de saúde da } \\
\text { população }\end{array}$ \\
\hline \multirow{3}{*}{$\begin{array}{l}\text { As mudanças no processo de trabalho em } \\
\text { equipe, na perspectiva dos elementos da } \\
\text { EIP, desencadeadas pelos ciclos formativos }\end{array}$} & $\begin{array}{l}\text { Para os médicos: mudança de postura em prol do melhor funcionamento } \\
\text { da equipe }\end{array}$ \\
\hline & $\begin{array}{l}\text { Para os formadores: redução de conflitos e potencialização dos processos } \\
\text { de comunicação }\end{array}$ \\
\hline & Para os profissionais de saúde: trabalho em equipe mais integrado \\
\hline \multirow{3}{*}{$\begin{array}{l}\text { Dificuldades para a abordagem da EIP nos } \\
\text { ciclos formativos }\end{array}$} & Formação uniprofissional \\
\hline & Teoria versus prática \\
\hline & Apoio aos formadores \\
\hline
\end{tabular}

Fonte: Elaboração própria.

\section{Os pressupostos da EIP enunciados nos ciclos formativos do PMM}

Os resultados desta categoria evidenciaram que alguns elementos teórico-metodológicos da EIP se enunciam nos processos pedagógicos desenvolvidos no âmbito do eixo do provimento emergencial do PMM, entre eles o aprendizado sobre o papel de cada profissão, considerado com um dos pressupostos centrais para a superação da hierarquia profissional e da atenção centrada no médico:
Ajudou muito para definir as funções de cada profissional, dos agentes comunitários, do enfermeiro e qual é nossa ligação com eles. (Médico intercambista).

Facilitou bastante porque eu consegui ver a função de cada profissional no meu âmbito de trabalho, para a gente conseguir facilitar, para que o trabalho evoluísse. (Médico brasileiro).

A modificação das práticas de cuidado a partir da colaboração profissional foi 
identificada como imprescindível para a melhoria dos resultados de saúde, tal como enuncia o objetivo central da EIP. Há o reconhecimento da relevância do trabalho em equipe para o enfrentamento adequado das necessidades e problemas de saúde dos usuários, família e comunidade:

Uma das preocupações é ensinar aos profissionais de saúde que o trabalho em equipe é de grande importância para melhorar a saúde da população. As pessoas que trabalham em equipe têm maior resolutividade das questões que tem atendido diariamente. (Médico cubano).

Deixa o serviço mais eficiente, melhora a satisfação do usuário. [...] Nem todo o profissional sai com essa visão da importância da multidisciplinaridade, interação entre os profissionais. (Médico brasileiro).

As práticas profissionais colaborativas estimuladas no âmbito dos ciclos formativos incluem as reuniões em equipe, o compartilhamento de experiências e a construção de planos de cuidados conjuntos: "Nos nossos encontros locorregionais a gente tenta trabalhar essa questão de troca de experiências, de ensinar e aprender" (Tutor).

É bastante incentivada a formação de grupos educativos, nos quais eles devem não apenas trabalhar a educação em saúde, mas também ajudar na formação e capacitação de ACS. [...] $E$ eu vi que alguns médicos, especialmente os cubanos, tentam incorporar essa sistemática, em que eles conversam e capacitam enfermeiros, técnicos de enfermagem e ACS. (Supervisor).

No curso tinha módulos que estimulavam no sentido de dar algumas ideias do que a gente poderia fazer na prática, construção de conhecimento, grupos operativos, dar 'feedback' para equipe do que estava sendo aprendido. (Médico brasileiro).

Meu supervisor também estimula a apresentar casos e a importância do trabalho em equipe, como é que funciona quando todos têm um comum acordo. (Médico intercambista).

Em toda supervisão eu pergunto como está a prática, se estão fazendo grupos com outros profissionais, se estão fazendo visita domiciliar junto com a enfermagem. [...] e é aí que a gente consegue identificar as falhas, e tenta estimular, incentivar para ver se surte algum efeito. [...] Muitas vezes a gente chama a enfermeira para conversar, para ver se a gente consegue estruturar de forma mais adequada a atenção primária em cada lugar, mas é um grande desafio. (Supervisor).

Os profissionais compreendem as necessidades das pessoas, famílias e comunidade como elemento ordenador do nível de interação entre os profissionais e da própria dinâmica do trabalho em saúde. Essa percepção se aproxima do pressuposto de aprendizagem compartilhada baseada nas necessidades de saúde da população, elemento da EIP que ratifica o paciente como sujeito central, e não como objeto do cuidado de saúde:

Fomos estimulados a conhecer a cidade e o município em que estávamos inseridos, a realidade, a sair para a rua e ver os problemas, falar com as referências do bairro. (Médico intercambista).

Para finalizar o curso de especialização a gente apresentou um TCC [trabalho de conclusão de curso]. E ele dependia do trabalho em equipe, identificação dos problemas... não foi eu que defini qual o problema a discutir e trabalhar na comunidade. Foi identificado pela comunidade e pelos integrantes da equipe. (Médico cubano).

Quando nós começamos a desenvolver diagnóstico de saúde da área de abrangência, tivemos que trabalhar com os agentes de saúde, com enfermeiro, estudantes de enfermagem, que nos ajudaram muito. Isso nos estimulou a fazer um trabalho em equipe, muitas opiniões, chuvas de ideias e que ao final foi o que nos ajudou a saber os principais problemas de saúde da população. (Médico cubano). 


\section{As mudanças no processo de traba- lho em equipe, na perspectiva dos elementos da EIP, desencadeadas pelos ciclos formativos}

Esta categoria apresenta as apreensões de mudanças, enunciadas pelos atores do PMM, que se relacionam com os pressupostos da EIP. Verifica-se que, nas falas dos diferentes atores - médicos, formadores e profissionais da ESF -, sobressaiu uma perspectiva distinta, mas que, confluídas, conduzem a um mesmo desfecho: maior colaboração profissional no cotidiano do trabalho em saúde.

Na perspectiva de alguns médicos, os conhecimentos adquiridos por meio dos ciclos formativos desencadearam certa mudança de postura, por parte deles, ante os demais profissionais da equipe:

Começamos a fazer reuniões regulares com a equipe. (Médico brasileiro).

Mudou a gestão do trabalho. Facilitou bastante porque eu consegui ver a função de cada profissional no meu âmbito de trabalho, como cada um atua para que o trabalho evolua. (Médico brasileiro).

Do ponto de vista dos tutores e supervisores, o maior impacto se relaciona com a resolução de conflitos entre os profissionais integrantes das equipes:

Contribuiu para reforçar o trabalho em si, a importância de cada profissional para o trabalho em equipe. (Supervisor).

A gente conseguiu desenvolver melhor a questão do trabalho em equipe, mostrando perspectivas diferentes, modalidades de trabalho em equipe diferentes, saber entender o outro dentro das suas dificuldades, que é um dos grandes problemas do trabalho em equipe. Eu acho que isso meIhorou bastante. (Tutor).

Na perspectiva dos profissionais de saúde que atuam com os médicos do PMM, as mudanças percebidas estão associadas ao perfil profissional do médico, e não ao programa especificamente. Nesse sentido, eles consideram que os médicos estrangeiros, em especial, os cubanos, são mais sensíveis ao trabalho colaborativo:

No meu entendimento, a gente aprendeu muita coisa com ele e ele aprendeu muita coisa com a gente. (Equipe de ESF - perfil cubano).

Você tem a possibilidade de trocar mais com o médico, de trabalhar mais junto... ele não chega só para atender e ir embora. Ele troca experiência, escuta seu ponto de vista e discute alguns casos. (Equipe de ESF - perfil cubano).

Eu acho que tem uma diferença muito grande no relacionamento. Eles [cubanos] são mais abertos, mais acessíveis. Não tem tanto aquele negócio 'eu sou médico, por favor, não fale comigo'. É mais fácil de chegar, não temos receio de perguntar e discutir casos. (Equipe de ESF - perfil cubano).

Tem a tutora que comparece e sempre procura informações com os outros profissionais sobre $o$ trabalho do médico, fazendo a gente se envolver em trabalhos de equipe, juntar as experiências do enfermeiro com o médico para tentar trazer resoluções para os pacientes. (Equipe de ESF - perfil brasileiro).

Na fala dos profissionais, também se enuncia que a ausência do médico limita o trabalho da ESF; e, nesse sentido, o PMM torna-se oportuno por assegurar a completude das equipes de atenção básica.

$O$ ponto forte é $O$ atendimento integral. $O$ agente comunitário fala do diagnóstico, fala do ambiente, das vulnerabilidades sociais. Eu com a minha percepção, a auxiliar com a dela, o médico com a dele. A gente consegue. [...] Quando a gente une a equipe toda, cada um dando uma opinião, a gente consegue fazer alguma coisa, pelo menos 
momentaneamente para tentar sanar a necessidade ou a fragilidade naquele momento. (Equipe de ESF - perfil intercambista).

\section{Dificuldades para a abordagem da EIP nos ciclos formativos}

Esta categoria compreende os pontos de fragilidade dos ciclos formativos que atuam como intervenientes para a adoção de ações pedagógicas alicerçadas na EIP. Entre eles - e, talvez, o mais importante -, está o fato de os ciclos formativos do PMM incluírem, formalmente, apenas os médicos nos processos de formação. Essa opção vai de encontro ao preceito fundamental da EIP de aprendizado entre duas ou mais profissões, e reforça a lógica de formação uniprofissional e de verticalidade hierárquica das relações profissionais:

Eu acho que o curso de especialização deveria ser interdisciplinar, seria mais rico o ambiente de discussão, porque cada um poderia trazer a experiência da própria área. (Médico brasileiro).

Eu não me lembro de termos feito, nesses três anos de programa, nenhum processo de formação que envolvesse formalmente outros profissionais da equipe. (Supervisor).

O coordenador [da equipe] tem que ser o médico, porque a gente fica chateado... uma pessoa chega e fala que não é assim e tem que mudar, uma pessoa que está num nível... não é desfazendo, mas como uma enfermeira vai questionar o meu trabalho? Isso é uma coisa absurda. (Médico cubano).

Ficou evidenciado que os elementos da EIP, que se enunciam sobremaneira a partir dos processos teóricos de formação, são pouco aplicados e discutidos no contexto prático. Essa frágil integração entre teoria e prática dificulta o desenvolvimento de competências colaborativas.
Poderia ser um pouco mais prático, porque a gente não abordou tanto as funções de cada um ou o trabalho em equipe nas avaliações com o supervisor; se poderia mostrar alguma dinâmica, algo que mostre para a gente como funciona na prática. (Médico intercambista).

A formação em saúde, especialmente em se tratando dos processos de educação permanente, por sua complexidade, exige a adoção de referenciais e métodos pedagógicos dinâmicos e alicerçados na aprendizagem significativa e na realidade dos serviços. Por outra via, a exigência do uso de tais métodos pelos formadores implica capacitá-los para tal e garantir-lhes condições e espaços para o desenvolvimento das ações educativas:

O PMM pede que utilizemos várias metodologias para fazer a supervisão, mas nem sempre a gente conhece todas elas. Então, poderíamos ser capacitados para entender as metodologias para que possamos usar métodos diferentes dos usuais. (Supervisor).

A supervisão não é considerada dentro das $8 \mathrm{~h}$ destinadas para a educação permanente dos médicos, o que significa que tivemos que fazer as supervisões dentro do horário de trabalho, o que traz impacto para a assistência. (Supervisora).

Eu acredito que se tivéssemos um referencial teórico para trabalhar isso, se fossemos treinados para desenvolver um trabalho de educação permanente visando uma melhor interação multiprofissional, com certeza teríamos bons resultados. Mas nós nunca fomos cobrados e nem nunca nos foi passado que esta abordagem deveria ser realizada. (Supervisor).

\section{Discussão}

O provimento médico se configura como política estratégica de melhoria da qualidade da atenção à saúde diante do cenário 
de desigualdade na distribuição inter e intrarregional desse profissional no País. Essa desigualdade decorre, sobremaneira, da dificuldade de fixar médicos em áreas remotas ou de vulnerabilidade social e em razão do modelo histórico de formação médica disciplinar, pouco comprometido com as transformações sociais e que tem valorizado a formação de especialistas em detrimento da formação de generalistas para atuação na atenção básica ${ }^{10-12}$.

É importante fazer essa ponderação para demarcar a importância do PMM no cenário atual brasileiro, que compartilha das mesmas dificuldades que o restante do mundo: hiato entre o perfil de formação e necessidades de saúde, limitada capacidade de análise do contexto; dificuldade para o trabalho em equipe e distribuição desigual dos profissionais de saúde nas diferentes regiões dos países ${ }^{13-15}$.

Pela importância da política é que se reconhece a capacidade de transformação do PMM e da coerência que o provimento venha acompanhado de iniciativas educacionais, uma vez que a mudança na lógica do trabalho precisar vir acompanhada de processos de mudanças na formação dos profissionais de saúde. É nesse entendimento que reside a necessidade de sistematizar o uso dos princípios da EIP nos ciclos formativos do PMM, abordagem pedagógica que vem sendo amplamente discutida em todo o mundo dada a sua capacidade de melhorar, em longo prazo, a qualidade dos cuidados ${ }^{16,17}$.

Partindo do pressuposto de que a EIP se configura como a ocasião em que diferentes profissões da saúde aprendem de forma conjunta, interativamente, com a clara intencionalidade de melhorar o processo de trabalho e a qualidade da atenção à saúde ${ }^{18}$, é possível afirmar que o propósito primeiro dessa abordagem é o desenvolvimento das competências colaborativas ${ }^{19}$. No entanto, juntar, em um mesmo espaço, pessoas de diferentes profissões não é suficiente para o desenvolvimento dessas competências.

É preciso assumir a intenção de formar profissionais de saúde mais aptos à colaboração para o verdadeiro trabalho em equipe, indispensável para o atendimento das complexas necessidades de saúde. Essa intencionalidade implica a organização sistematizada de conteúdos e de métodos de ensino e aprendizagem, teóricos e práticos, que estimulem o desenvolvimento de capacidades profissionais cooperativas ${ }^{20}$.

As competências colaborativas são definidas como aquelas que viabilizam o trabalho em equipe efetivo, resolutivo e alinhado às necessidades de saúde ${ }^{21}$. A produção científica apresenta contribuições valorosas que podem colaborar para o reconhecimento, construção e/ou ajuste dessas competências, em consonância ao contexto em que a EIP é operada.

As publicações mais conhecidas no cenário internacional são as matrizes de competências do Canadá22 e dos $\mathrm{EUA}^{23}$, que servem como guia para o desenvolvimento de currículos de processos de formação na saúde. Ambas ratificam a importância do reconhecimento do papel do outro como pressuposto para melhorar a interação, reconhecendo o caráter complementar e interdependente entre as diferentes categorias profissionais.

Sobre isso, neste estudo, ficou evidenciado que os domínios centrais apresentados nas referidas matrizes ${ }^{\mathbf{2 2 , 2 3}}$ são abordados nos ciclos formativos do PMM: o reconhecimento do papel do outro; o funcionamento da equipe; a comunicação interprofissional; e o reconhecimento da necessidade de (re)situar as demandas dos usuários, famílias e comunidade como núcleo do processo de trabalho em saúde.

A centralidade no usuário e nas suas demandas, característica precípua das práticas colaborativas, concretiza-se por ocasião de maior envolvimento da equipe nos processos de discussão e planejamento das ações de enfrentamento dos problemas de saúde do território, que exige, por sua vez, o reconhecimento de objetivos comuns entre os profissionais de saúde. A partir desse reconhecimento, a equipe identifica o papel e a contribuição de cada categoria, melhorando o nível de interação em um movimento de comunicação mais fluido, que tem como desdobramentos a melhoria da 
qualidade da atenção à saúde e o aumento da satisfação do usuário ${ }^{24}$.

Outro aspecto que merece destaque nos resultados encontrados por este estudo é que a política de provimento médico possibilitou a reversão de cenários de saúde, nos quais as equipes de ESF, incompletas pela ausência desse profissional, estavam consideravelmente limitadas para promover atenção integral às populações ${ }^{15}$. A análise das situações de saúde sob a perspectiva de diferentes profissionais - olhares que se somam - assegura uma compreensão mais aprofundada dos determinantes de saúde e, ao mesmo tempo, permite atuações interdisciplinares e interdependentes ampliadas ${ }^{25}$. Nesse prisma, a interprofissionalidade constitui-se estratégia catalisadora para a mudança do modelo de atenção à saúde.

Segundo as falas dos participantes, parceria, interdependência, compartilhamento e resolução de conflitos são fundamentos da colaboração profissional ${ }^{\mathbf{2 6}, 27}$ enunciados nos ciclos formativos do PMM, embora o exercício para o desenvolvimento dessas competências não tenha sido completamente assegurado pelas estratégias pedagógicas, em especial, no campo prático. Delineia-se que, em grande medida, esses princípios ancoram a proposta de trabalho da ESF, cabendo aos processos formativos o fortalecimento dessa abordagem.

Embora o PMM se apresente como espaço potente para a EIP, ainda há importantes lacunas que, uma vez preenchidas, podem conduzir o programa a alcançar de maneira mais efetiva os seus objetivos. Entre elas, a que mais chama a atenção é o direcionamento das ofertas educacionais apenas aos médicos. É bem verdade que a política assume como proposta mudar a lógica do trabalho e formação médica para uma que se alinhe às necessidades do SUS. Entretanto, essa mudança só pode ser inteiramente concretizada se dela participarem todos os atores imbricados na produção do cuidado.

Ademais, o aprendizado conjunto é condição sine qua non para a superação das manifestações hegemônicas de poder e dos silos profissionais, que se ancoram, grosso modo, na lógica da fragmentação das áreas de conhecimento ${ }^{\mathbf{2 8}}$, dispondo-se, portanto, contrariamente ao princípio da integralidade.

Este estudo identificou, ainda, que o PMM estimula os formadores - supervisores e tutores - a adotarem métodos pedagógicos inovadores, tal como prevê a EIP, segundo a qual são essenciais para o aprendizado compartilhado e interativo ${ }^{29}$. Contudo, o estímulo não é acompanhado de processos de desenvolvimento docente, como pontuado pelos entrevistados.

Assumindo o desenvolvimento docente como um conjunto de ações empregadas para qualificar o corpo docente sobre seus papeis, de forma a potencializar os processos de formação, ele se faz nuclear para a transformação das práticas pedagógicas e, por conseguinte, das práticas laborais em saúde. Para a EIP, o docente atua como mediador nas situações de aprendizagem, competência esta que demanda por desenvolvimento dos atores formativos, situando-o como um eixo central diante das inovações educacionais, da potência dos cenários de prática como espaços seguros e profícuos de compartilhamento de saberes e do complexo processo de discussão dos papeis profissionais ${ }^{\mathbf{3 0}, 31}$.

O comprometimento com uma formação baseada nos pressupostos da interprofissionalidade, no entanto, não pode ser reduzida à figura do formador. É requerido apoio institucional - das universidades, serviços de saúde e Estado -, que estimulem a criação de uma cultura de formação e trabalho em saúde integradora, colaborativa e interprofissional ${ }^{32,33}$.

Pensar estratégias de fortalecimento da EIP no PMM é um caminho importante, e necessário, para que a política seja sustentável e para que os resultados almejados sejam alcançados e solidificados. As falas dos participantes apontam que a dinâmica do trabalho em saúde na ESF é favorável para o desenvolvimento das competências colaborativas. O grande salto será possível quando os princípios da EIP puderem ser mais bem incorporados nos itinerários formativos do PMM. 


\section{Considerações finais}

O estudo evidenciou que alguns elementos centrais da EIP se enunciam nos processos formativos desenvolvidos no âmbito do eixo do provimento emergencial do PMM, a exemplo do aprendizado sobre o papel de cada profissão, o compartilhamento de experiências e a centralidade do cuidado no paciente. Por outra via, o preceito fundamental da EIP de aprendizado entre duas ou mais profissões não é aplicado sistematicamente, dado que os ciclos formativos se destinam, formalmente, apenas aos médicos do programa. Tal fato, no entanto, não exclui a possibilidade de desenvolvimento das competências colaborativas, a partir da inclusão das diferentes categorias profissionais nos processos de formação operados nos cenários de produção do cuidado em saúde.

O Mais Médicos configura-se como um espaço adequado para a EIP, na medida em que assegura a conformação mínima da equipe de ESF, oportunizando, assim, uma dinâmica de trabalho integralizada. Nesse cenário, a introdução intencional e sistemática dos princípios da EIP, para que as competências colaborativas se desenvolvam, pode repercutir sobremaneira na melhoria da qualidade da atenção - que é o horizonte maior dessa estratégia.

Isto exposto, depreende-se que o PMM se constitui política potente e propícia para o fortalecimento da interprofissionalidade, especialmente porque sua proposta se assenta na lógica da educação permanente, o que demanda o estabelecimento de uma relação de mútua influência entre o campo da educação e do trabalho em saúde.

Considerando o PMM enquanto política recente e a incipiência de experiências documentadas sobre a EIP no contexto nacional, torna-se imperativo estabelecer um processo permanente de reflexão e avaliação dessas propostas. Nessa perspectiva, mais estudos são necessários para testar a influência delas sobre o trabalho em equipe e, por conseguinte, sobre a capacidade de resposta às demandas de saúde da população.

Dadas as fragilidades constatadas nos ciclos formativos que se relacionam aos elementos teórico-metodológicos da EIP que obstam a prática colaborativa, recomenda-se a construção de uma oferta educativa sobre a EIP e colaboração profissional, para ser disponibilizada aos médicos do provimento emergencial do PMM e, oportunamente, aos demais integrantes das equipes de ESF. Vê-se, nessa proposta, uma oportunidade estratégica de apresentar e consolidar o conceito de EIP e de estimular o desenvolvimento das competências e das práticas colaborativas no cotidiano do trabalho em saúde.

\section{Colaboradores}

Freire Filho JR (0000-0003-1306-9368)* contribuiu para a concepção, planejamento, análise e interpretação dos dados; para a elaboração do rascunho, revisão crítica do conteúdo e aprovação final do manuscrito. Magnago C (0000-0001-8799-3225)* contribuiu para a concepção, planejamento, análise e interpretação dos dados; para a elaboração do rascunho, revisão crítica do conteúdo e aprovação final do manuscrito. Costa MV (00000002-3673-2727)* contribuiu para a análise e interpretação dos dados; para a elaboração do rascunho, revisão crítica do conteúdo e aprovação final do manuscrito. Forster AC (0000-0002-2720-5802)* contribuiu para a concepção e planejamento da proposta, revisão crítica e aprovação da versão final. 


\section{Referências}

1. World Health Organization. Framework for action on interprofessional education and collaborative practice. Geneva; Switzerland: World Health Organization; 2010.

2. World Health Organization. WHO education guidelines: transforming and scaling up heath professionals' education and training. Geneva; Switzerland: World Health Organization; 2013.

3. Frenk J, Chen L, Bhutta ZA, et al. Health professionals for a new century: transforming education to strengthen health systems in an interdependent world. Lancet [internet]. 2010 [acesso em $2018 \mathrm{abr}$ 12]; 376(9756):1923-58. Disponível em: https://doi. org/10.1016/S0140-6736(10)61854-5.

4. Centre for the Advancement of Interprofessional Education - CAIPE. Interprofessional Education Guidelines 2017. United Kingdom: CAIPE; 2017.

5. Brasil. Ministério da Educação. Resolução $n^{\circ} 3$, de 20 de junho de 2014. Institui Diretrizes Curriculares Nacionais do Curso de Graduação em Medicina e dá outras providências. Brasília, DF: Diário Oficial da União. 23 Jun 2014.

6. Freire Filho JR, Costa MV, Forster AC, et al. New national curricula guidelines that support the use of interprofessional education in the Brazilian context: an analysis of key documents. J. Interprof. Care [internet]. 2017 [acesso em 2018 abr 18]; 31(6):754-60. Disponível em: https://doi.org/10.1080/13561820.20 17.1346592 .

7. Freire Filho JR, Magnago C, Costa MV, et al. Cursos de especialização ofertados no âmbito do Mais Médicos: análise documental na perspectiva da Educação Interprofissional. Interface (Botucatu) [internet]. 2018 [acesso em 2018 abr 18]; 22(sup2):1613-1624. Disponível em: http://dx.doi. org/10.1590/1807-57622017.0842.

8. Bardin L. Análise de Conteúdo. Lisboa: Edições 70; 2011.
9. Barr H. Ensuring quality in interprofessional education. CAIPE Bulletin. 2003; (22):2-3.

10. Campos FE, Machado MH, Girardi SN. A fixação de profissionais de saúde em regiões de necessidades. Divulg. Saúde Debate. 2009; (44):13-24.

11. Bispo Junior JP, Moreira DC. Educação permanente e apoio matricial: formação, vivências e práticas dos profissionais dos Núcleos de Apoio à Saúde da Família e das equipes apoiadas. Cad. Saúde Pública [internet]. 2017 [acesso em 2018 abr 18); 33(9):e00108116. Disponível em: http://dx.doi. org/10.1590/0102-311X00108116.

12. Dal Poz MR, Perantoni CR, Girardi S. Formação, mercado de trabalho e regulação da força de trabalho em saúde no Brasil. In: Fundação Oswaldo Cruz. A saúde no Brasil em 2030 - prospecção estratégica do sistema de saúde brasileiro: organização e gestão do sistema de saúde. Rio de Janeiro: Fiocruz/Ipea/Ministério da Saúde/Secretaria de Assuntos Estratégicos da Presidência da República; 2013. v. 3. p. 187-233.

13. Dolea C, Stormont L, Braichet JM. Evaluated strategies to increase attraction and retention of health workers in remote and rural areas. Bull. World Health Organ. [internet]. 2010 [acesso em $2018 \mathrm{abr}$ 18]; 88(5):379-385. Disponível em: http://dx.doi. org/10.2471/BLT.09.070607.

14. Rourke J. WHO recommendations to improve retention of rural and remote health workers - important for all countries. Rural Remote Health 2010; 10(4):1654.

15. Santos LMP, Costa AM, Girardi SN. Mais Médicos Program: an effective action to reduce health inequities in Brazil. Ciênc. Saúde Coletiva [internet]. 2015 [acesso em 2018 abr 14]; 20(11):3547-52. Disponível em: http:// dx.doi.org/10.1590/1413-812320152011.07252015.

16. Schmitt MH, Gilbert JH, Brandt BF, et al. The coming of age for interprofessional education and practice. 
Am. J. Med. [internet]. 2013 [acesso em 2018 abr 14]; 126(4):284-288. Disponível em: http://dx.doi. org/10.1016/j.amjmed.2012.10.015.

17. Barr H. Interprofessional Education: the genesis of a global movement. United Kingdon: Center For The Advancement of Inteprofessional Care; 2015.

18. Reeves S, Perrier L, Goldman J, et al. Interprofessional education: effects on professional practice and healthcare outcomes (update). Cochrane Database Syst. Rev. [internet]. 2013 [acesso em 2018 abr 17]; 28(3):CD002213. Disponível em: https://doi. org/10.1002/14651858.CD002213.pub3.

19. Cuff P, Schmitt M, Zierler B, et al. Interprofessional education for collaborative practice: views from a global forum workshop. J. Interprof. Care [internet]. 2014 [acesso em 2018 abr 17]; 28(1):2-4. Disponível em: https://doi.org/10.3109/13561820.2013.828910.

20. Reeves S. Ideas for the development of the interprofessional education and practice field: an update. J. Interpr. Care [internet]. 2016 [acesso em 2018 abr 17]; 30(4):405-407. Disponível em: https://doi.org/10.10 80/13561820.2016.1197735.

21. Barr H. Competent to collaborate: towards a competency-based model for interprofessional education. J. Interpr. Care [internet]. 1998 [acesso em 2018 abr 17]; 12(2):181-187. Disponível em: https:// doi.org/10.3109/13561829809014104.

22. Canadian Interprofessional Health Collaborative. A National Interprofessional Competency Framework. Vancouver: CIHC; 2010.

23. Interprofessional Education Collaborative. Core competencies for interprofessional collaborative practice: 2016 update. Washington, DC: Interprofessional Education Collaborative; 2016.

24. Xyrichis A, Reeves S, Zwarenstein M. Examining the nature of interprofessional practice: an initial framework validation and creation of the InterProfessional Activity Classification Tool (InterPACT). J. Interpr. Care [internet]. 2017 [acesso em 2018 jun 3]; 13:1-10. Disponível em: https://doi.or g/10.1080/13561820.2017.1408576.

25. Viegas SMF, Penna CMM. A construção da integralidade no trabalho cotidiano da equipe saúde da família. Esc. Anna Nery [internet]. 2013 [acesso 2018 jun 3]; 17(1):133-141. Disponível em: http://dx.doi. org/10.1590/S1414-81452013000100019.

26. D’Amour D, Ferrada-Videla M, San Martin Rodriguez $\mathrm{L}$, et al. The conceptual basis for interprofessional collaboration: core concepts and theoretical frameworks. J. Interprof. Care [internet]. 2005 [acesso em 2018 jun 4]; 19(supl):116-131. Disponível em: http:// dx.doi.org/10.1080/13561820500082529.

27. San Martín-Rodríguez L, Beaulieu MD, D’Amour D, et al. The determinants of successful collaboration: a review of theoretical and empirical studies. J. Interprof. Care [internet]. 2005 [acesso em 2018 jun 4]; 19(supl):132-147. Disponível em: http://dx.doi. org/10.1080/13561820500082677.

28. Weller J. Shedding new light on tribalism in health care. Med. Educ. [internet]. 2012 [acesso 2018 jun 4]; 46(2):134-136. Disponível em: http://dx.doi. org/10.1111/j.1365-2923.2011.04178.x.

29. Peduzzi M. The SUS is interprofessional. Interface (Botucatu) [internet]. 2016 [acesso em 2018 jun 12]; 20(56):199-201. Disponível em: http://dx.doi. org/10.1590/1807-57622015.0383.

30. Steinert Y, Mann K, Centeno A, et al. A systematic review of faculty development initiatives designed to improve teaching effectiveness in medical education: BEME Guide No. 8. Med. Teac. [internet]. 2006 [acesso em 2018 jun 16]; 28(6):497-526. Disponível em: http://dx.doi.org/10.1080/01421590600902976.

31. Batista NA, Batista SHSS. Interprofessional education in the teaching of the health professions: shaping practices and knowledge networks. Interface (Botucatu) [internet]. 2016 [acesso 2018 jun 12]; 20(56):202-204. Disponível em: http://dx.doi. org/10.1590/1807-57622015.0388. 
32. Reeves S. Why we need interprofessional education to improve the delivery of safe and effective care. Interface (Botucatu) [internet]. 2016 [acesso em 2018 jun 12]; 20(56):185-97. Disponível em: http://dx.doi. org/10.1590/1807-57622014.0092.

33. Barros NF, Spadacio C, Costa MV. Trabalho interprofissional e as Práticas Integrativas e Complementares no contexto da Atenção Primária à Saúde: potenciais e desafios. Saúde debate [internet]. 2018 [acesso em 2019 mar 12]; 42(esp1):163-173. Disponível em: http:// dx.doi.org/10.1590/0103-11042018s111.

Recebido em 09/08/2018

Aprovado em 13/03/2019

Conflito de interesses: inexistente

Suporte financeiro: não houve 